\title{
Arthroscopy Assisted Lesion Clearance and Bone Graft, Titanium Rod Support Treatment of Early Stage Osteonecrosis of the Femoral Head of the Postoperative Survival Rate Analysis
}

\author{
Xingming Yang1*, Wei Shi², Yakun Du3 ${ }^{3}$, Lei Zhang4 \\ ${ }^{1}$ Department of Orthopaedics, The First Affiliated Hospital of Hebei North University, Zhangjiakou, China \\ ${ }^{2}$ Department of Paediatrics, The First Affiliated Hospital of Hebei North University, Zhangjiakou, China \\ ${ }^{3}$ Department of Neurology, The Children's Hospital of Hebei Province, Shijiazhuang, China \\ ${ }^{4}$ Department of Neurosurgery, The 4th Affiliated Hospital of Hebei Medical University, Shijiazhuang, China \\ Email: *yxm1120@sohu.com
}

How to cite this paper: Yang, X.M., Shi, W., Du, Y.K. and Zhang, L. (2017) Arthroscopy Assisted Lesion Clearance and Bone Graft, Titanium Rod Support Treatment of Early Stage Osteonecrosis of the Femoral Head of the Postoperative Survival Rate Analysis. Surgical Science, 8, 37-46.

http://dx.doi.org/10.4236/ss.2017.81005

Received: September 12, 2016

Accepted: January 10, 2017

Published: January 13, 2017

Copyright $\odot 2017$ by authors and Scientific Research Publishing Inc. This work is licensed under the Creative Commons Attribution International License (CC BY 4.0).

http://creativecommons.org/licenses/by/4.0/

(c) $\underset{\mathrm{EY}}{\mathrm{i}}$ Open Access

\begin{abstract}
Objective: To assess the curative effect of the stage II femoral head necrosis treated by arthroscopy assisted lesion clearance, bone graft and titanium rod support. Methods: All the patients (including 58 patients 74 hips) were diagnosed as stage II femoral head necrosis according to the ARCO staging system during 2003-2013. In these patients, 15 hips were stage IIA, 34 hips were stage IIB and 25 hips were stage IIC. Located by $\mathrm{C}$-arm and assisted by arthroscopy, minimally-invasive percutaneous pulp core decompression and lesion clearance within the femoral head were accurately performed, and then, the OAM composite of autologous bone marrow was implanted and the femoral head was supported using the titanium rod. Follow-up including the pain score, the Harris hip score and X-ray observation for disease progression were achieved at 6,12, 24 and 36 months postoperatively, Kaplan-Meier survival curve was used for the survival analysis. Result: The VAS score and the Harris score after operation were better THRAn THRAt of before the surgery, the difference had statistical significance $(\mathrm{p}<0.05)$. As for the X-ray staging, 5 cases $(5 \mathrm{hips})$ progressed from stage IIB to stage IIC, the femoral head of 6 cases (6 hips) staged IIC collapsed at 24 months after the operation and then underwent THRA after 30 months. In this study, the total improvement rate after the surgery was $79.72 \%$ (93.33\% for IIA, $82.35 \%$ for IIB and $68 \%$ for IIC). The total survival rate of these patients was $64.2 \%$ (95\% CI, 64.2\% - 90.1\%). Conclusion: Arthroscopy assisted lesion clearance, bone graft and titanium rod support to treat the stage II osteonecrosis of the femoral head are effective and can prevent the femoral head from collapsing. But for stage IIC patients who had a history of the use of hormone, this surgery should be chosen carefully because the outcome is always very poor.
\end{abstract}




\section{Keywords}

Femur Head Necrosis, Survival Rate, Treatment Outcome

\section{Introduction}

As the incidence of osteonecrosis of the femoral head (ONFH) is increasing and the age of onset become younger, [1] [2] [3] [4], how to retain the femoral head and to prevent it from collapsing, avoiding the total hip replacement arthroplasty (THRA) too early, are developing into the trend of early treatment of ONFH [5] [6] [7]. Arthroscopy assisted percutaneous spinal core decompression and the implantation of osteoinductive absorbing material (OAM) which compounded with autologous red bone marrow (ARBM) associated with titanium rod support to treat the state II ONFH were widely accepted by the academic circle. Its early curative effect after the surgery is recognized, but the survival rate analysis of the femoral head after the surgery has not been reported statistically by any document. We used this method to treat early stage ONFH and achieved the complete follow-up data during the period of 2003.04 to 2013.12, making a survival analysis. The results are as follows.

\section{Methods}

\subsection{Design}

To evaluate the feasibility and clinical effect of the minimally invasive grafting osteoinductive absorbing material attaching autologous red bone marrow by the decompression of bone marrow core with the assistance of arthroscopy combined with Titanium rod in the treatment of stage II avascular necrosis of the femoral head.

\subsection{Time and Study Site}

The study was approved and granted by the Ethics Committee of the First Affiliated Hospital of Hebei North University and completed in the First Affiliated Hospital of Hebei North University in Zhangjiakou City, Hebei Province, China, between April of 2003 and December of 2013.

\subsection{Adoption Standard}

All the stage II ONFH patients caused by hormone, alcohol, trauma, and idiopathic diseases according to the Association Research Circulation Osseous (ARCO) classification [8].

\subsection{Elimination Standard}

The ONFH patients of stage I, III, IV according to ARCO classification, the patients of Immature bone development or those who have received immunosuppressive drugs, those who underwent inline fixation treatment for femoral neck fracture and those who present infection, now or past. 


\subsection{Subjects}

All of the 58 cases were consistent with the adoption standard, coming from Zhangjiakou of Hebei Province and six counties of Neimenggu in China, of which 28 cases from Zhangjiakou area (48.28\%) and 30 cases from Neimenggu areas $(51.72 \%)$. The ratio of male to female is 2.41:1, with male 41 cases, female 17 cases. Age Distribution: Maximum age is 53 years old, minimum age is 31 years old, 36 cases are $31-40$ years old, 18 cases are 41 - 50 years old, 4 cases are 51 - 53 years old, average age in this group is $38 \pm$ 3.5 years old. Diseased parts: There are 42 cases with sigular hip involved, of which 12 cases with left hip, 30 cases with right hip. There are 16 cases with double hip involved. The onset time: 10 to 28 months duration, an average of 18 months. Pathogenic causes and periodization: There are 28 cases with hormones cause, of which 6 cases with II A stage, 14 cases with IIB stage, 8 cases with IIC stage. There are 28 cases with alcohol causes, of which 4 cases with IIA stage, 14 cases with IIB stage, 10 cases with IIC stage. There are 10 cases with traumatic cause, of which 3 cases with II A stage, 3 cases with IIB stage, 4 cases with IIC stage. There are 8 cases with idiopathic cause, of which 2 cases with IIA stage, 3 cases with IIB stage, 3 cases with IIC stage. The pathogenic causes and corresponding ARCO clinical stage (Table 1).

\subsection{Surgical Method}

Type "C" arm X-ray machine perspective, under the femoral greater trochanter in $2 \mathrm{~cm}$ will be a $2 \mathrm{~mm}$ needle into central femoral head necrosis area (the positive side of X-ray fluoroscopy location right), the lateral incision hip, longitudinal form skin incision 1.5 $\mathrm{cm}$, separate muscularis, inserted into the diameter of $1.5 \mathrm{~cm}$ pipe work, in turn, with 6 , $8,10 \mathrm{~mm}$ hollow drill through the needle to the subchondral drilling area. In the $\mathrm{C}$ arm fluoroscopy and arthroscopy alternately under the guidance of the core decompression and bone tunnel with long handle high-speed grinding bone drill and spatula cleaning lesions, clear edge of bone tissue necrosis of femoral head were observed cystic lesion area and sclerotic bone are all curettage, or joint subchondral bone collapse whether pathological involvement the articular surface, or prevent the operation scraping through the joint surface, until the arthroscopy confirmed dead bone removal is thorough, close to normal trabecular bone and fresh blood oozing when the core decompression stop (Figures 1-4); with the hammering device through the tunnel will be implanted in the femoral head bone lesion curettage, appropriate compression the appropriate length of hollow titanium rod implanted into the femoral head, the incision was sutured.all operations are performed by the same performer.

All cases of implementation is made by authorization of the Hebei north college affi-

Table 1. 58 patients (74 hips) of pathogenic cause and ARCO stage (hip).

\begin{tabular}{ccccc}
\hline Cause & Hip number & IIA & IIB & IIC \\
\hline Cortine & 28 & 6 & 14 & 8 \\
Alcohol & 28 & 4 & 14 & 10 \\
Trauma & 10 & 3 & 3 & 4 \\
Idiopathetic & 8 & 2 & 3 & 3 \\
\hline
\end{tabular}




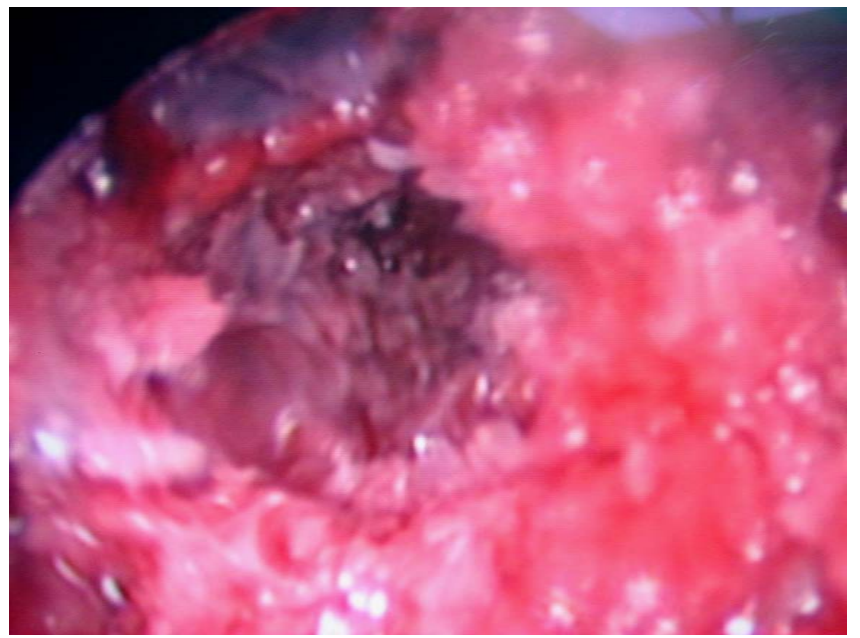

Figure 1. Observed the cystis degeneration and the ischemic necrotic bone tissues of the femoral head by the arthroscope.

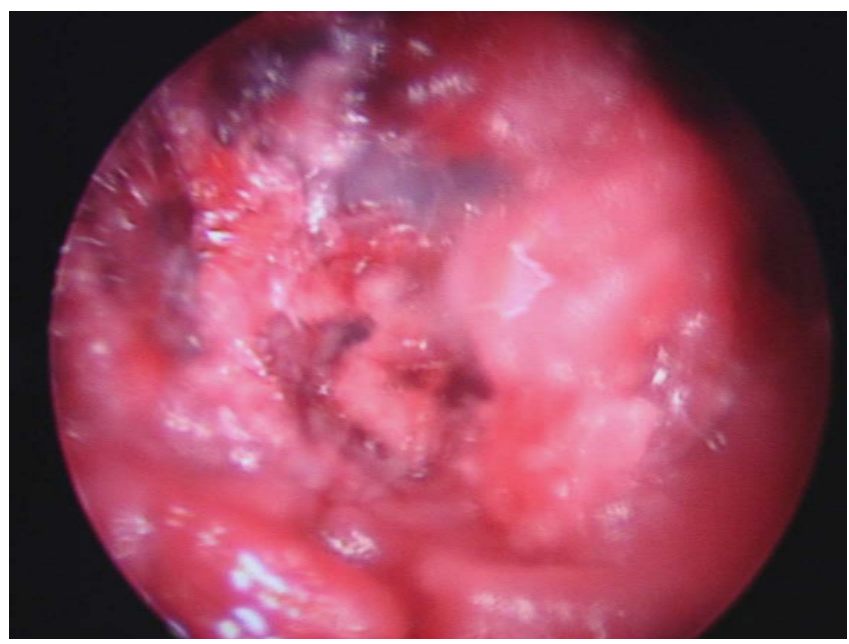

Figure 2. Observed the pathological, ischemic and loosed sclerotized bone of the femoral head by the arthroscope.

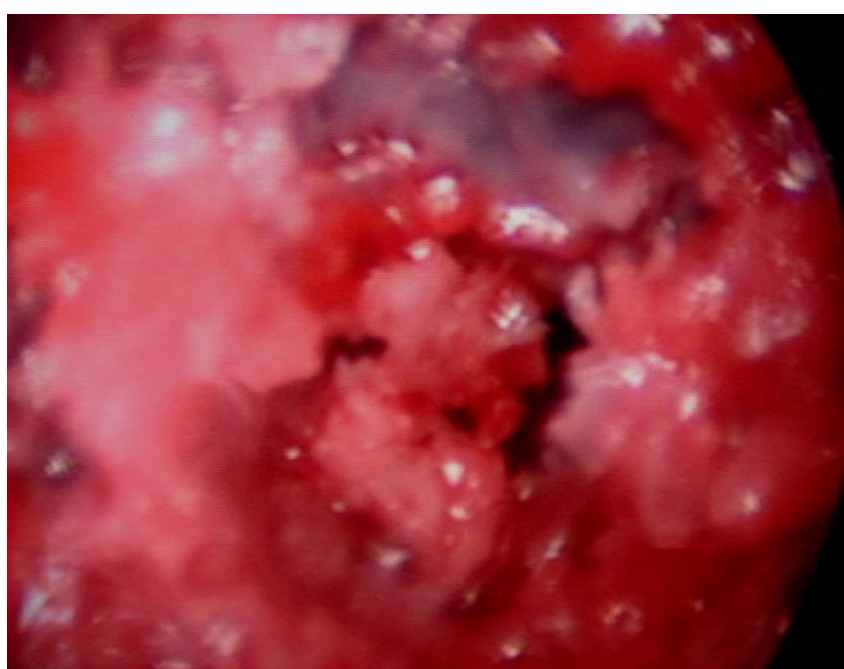

Figure 3. Observed the cystis degeneration accompanied with the ischemic sclerotized bone of the femoral head by the arthroscope. 


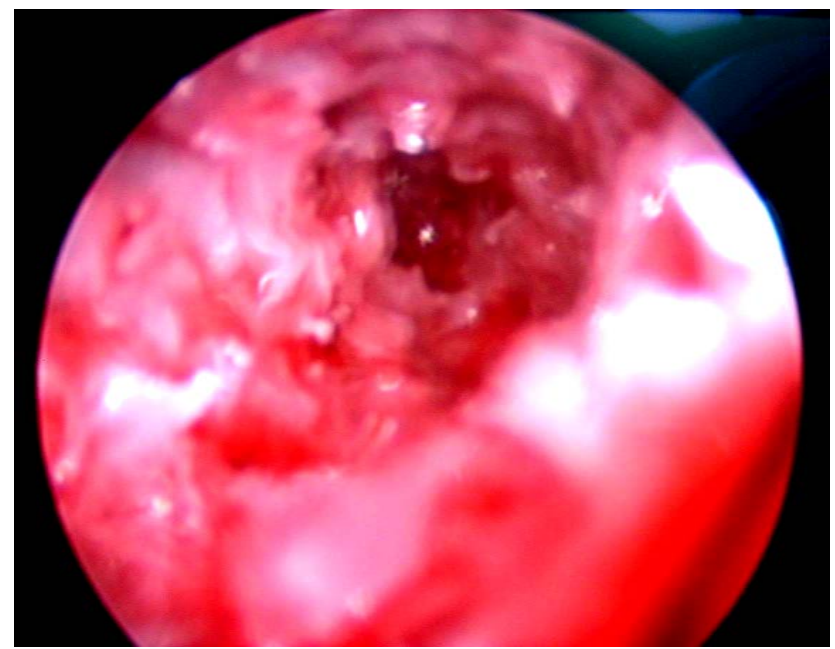

Figure 4. THRAt the blood supply was good after the pathological changes were cleared thoroughly was seen by arthroscope.

liated first hospital ethics committee, and inform the patient and family to sign a consent form.

\subsection{The Postoperative Recovery}

All patients should get a CPM exercise with hip and knee postoperatively. Single hip surgery patients should crutch with no weight-bearing activities until 3 months after surgery, then make a weight-bearing walking with single-throw gradually; Double hip surgery patients should rest in bed for at least 3 months, then walk helped with crutch for six months.

\subsection{Survival Evaluation Index}

The pain visual analogue score (VAS); to improve the function of the Harris hip score standard; X-ray observation of lesion stability: ARCO stage no change for stability, progress over the same period for a period of instability in Guinea, stage III or collapse of the femoral head is worse. The clinical curative effect to "improve" and "no change" and "aggravating" said: hip pain relief is more than 50\%, the daily activities of hip joint function improved more than $50 \%$, the X-ray manifestations of stability to "improve"; hip pain relief $<50 \%$, daily activities of hip joint function improved $<50 \%$, stable or unstable for X-ray manifestations "no change"; hip pain and hip joint function than before the treatment, the X-ray manifestations of deterioration aggravated". Survival analysis of hip arthroplasty (THRA) as a failure endpoint after lesion surgery.

\subsection{Analytical Method of the Data}

All the data were processed by SPSS13.5 software. multiple sets of measurement data using ANOVA. a $=0.05$ as Inspection level. The finish line for THRA observing is 36 months, Kaplan Meier survival curve are used for survival analysis.

\section{Results}

All cases in this group were followed up for 24 - 36 months (average of 32 months), 
pathological histology after surgery were proved as osteonecrosis.

\subsection{VAS, Harris Score Results before and after Operation}

Postoperative time points for 24 months and 12 months, in contrast, there was no statistically significant difference $(\mathrm{P}>0.05)$, and the rest of the time period before and after comparison, or compared with preoperative, VAS score or Harris scoring index improved significantly, the difference had statistical significance $(\mathrm{P}<0.05)($ Table 2$)$.

\subsection{X-Ray Stage Changes before and after Operation}

As for X-ray staging before and after surgery, five stage IIB patients progressed to IIC during 12 - 24 months after surgery, of which, 4 cases progress to stage III and underwent THRA at last. The original IIC phase has six hip femoral head collapse after 24 months, THRA after 30 months (Table 3).

\subsection{The Clinical Curative Effect of 36 Months Follow-Up after Operation}

Total improvement rate after the surgery was $79.72 \%$, IIA improvement rate $93.33 \%$ was best, IIB period improvement rate $82.35 \%$, IIC improvement rate $68 \%$ was lowest; no change and increase of cases are patients with glucocorticoid-induced ONFH (Table $4)$.

\subsection{Period Lesions Hip Survival Curves}

Based on the differences of etiology, stage II disease Kaplan-Meier survival curve, End to THRA (Figure 5). Eventually 36 months survival rate was $64.2 \%$ (95\% ci, 64.2\% $90.1 \%$ ), combined with what Tables $2-4$ shows IIC lesions 3 year survival rate was the lowest (Figure 5).

Table 2. Pain score and Harris hip score before and after the operation.

\begin{tabular}{cccc}
\hline Time & Hip number & Pain score & Harris score \\
\hline Before the treatment & 74 & $5.1 \pm 1.1(3-9)$ & $77.4 \pm 3.6(74-89)$ \\
6 months & 74 & $4.3 \pm 0.9(1-6)$ & $84.4 \pm 1.1(83-92)$ \\
12 months & 74 & $2.1 \pm 0.7(0-6)^{*}$ & $94.1 \pm 2.8(82-100)^{\star}$ \\
24 months & 74 & $2.1 \pm 0.5(0-10)^{*}$ & $93.9 \pm 2.7(80-100)^{\star}$ \\
36 months & 58 & $0.7 \pm 0.2(0-2)$ & $98.8 \pm 2.0(95-100)$ \\
& & $\mathrm{F}=144.11$ & $\mathrm{~F}=338.24$ \\
Statistic & - & $\mathrm{P}<0.05$ & $\mathrm{P}<0.05$
\end{tabular}

Compared with before treatment, the difference was statistically significant $(\mathrm{P}<0.05)$. ${ }^{*}$ Which shows no difference.

Table 3. X-ray staging changes before and after the operation.

\begin{tabular}{cccccc}
\hline \multicolumn{2}{c}{ Preoperative } & \multicolumn{4}{c}{ Postoperative stage } \\
Staging & Number hip & IIA & IIB & IIC & III \\
IIa & 15 & 15 & 0 & 0 & 0 \\
IIb & 34 & 0 & 29 & 1 & 4 \\
IIc & 25 & 0 & 0 & 19 & 6 \\
\hline
\end{tabular}


Table 4. The clinical curative effect of 36 months follow-up.

\begin{tabular}{ccccc}
\hline & Total & IIA & IIB & IIC \\
\hline Risk of hip number & 74 & 15 & 34 & 25 \\
Improved & 59 & 14 & 28 & 17 \\
No change & 4 & 1 & 1 & 2 \\
add & 11 & 0 & 5 & 6 \\
THRA & 10 & 0 & 4 & 6 \\
Improvement rate (\%) & 79.72 & 93.33 & 82.35 & 68 \\
\hline
\end{tabular}

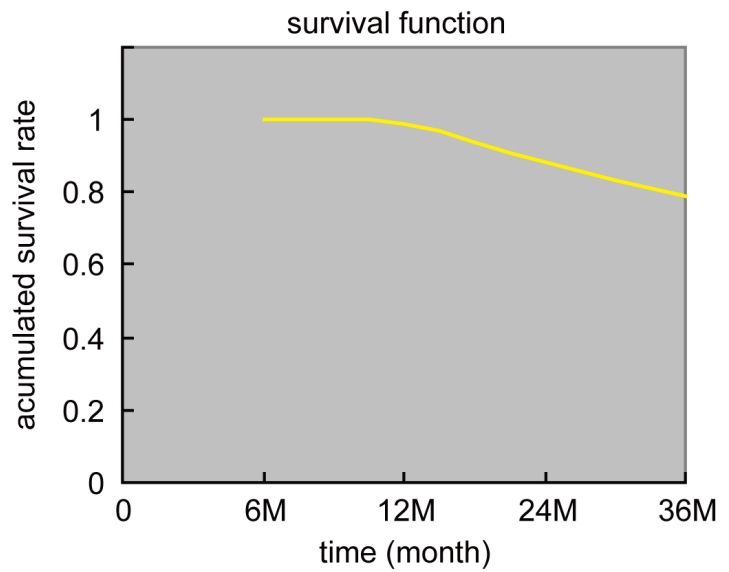

Figure 5. The Kaplan-Meier survival curves of II period lesions hip with End to THRA.

\section{Discussion}

There are many methods of treatment for ONFH in the clinical, but it is ideal in academic that THRAt save the patient's own femoral head and prevent joint surface subsidence for early ONFH. The optimal treatment time is in the early stage. Reducing the femoral medullary cavity pressure, removing the necrotic tissue, improving blood circulation within the femoral head, strengthening the repairing ability of the femoral head should be carried out to promote bone regeneration and restore the femoral head anatomy and tissue structure. Before the lesion repair, maintaining local mechanical strength, preventing collapse and delaying or preventing disease development should be considered. The design of this research is, by the arthroscope, removing necrosis lesions of femoral head, grafting bone to promote bone repair and using Titanium rod to maintain mechanical strength and prevent femoral head collapse, which conform to the therapy principles of ONFH. But the condition of some cases worsened and the femoral head collapsed two years after the operation during the clinical follow-up. Thus, how to improving the survival rate of the femoral head, ensuring clinical effect, analyzing the reasons of failure and formulating precise surgical incications are very necessary [9] [10] [11].

We find through the postoperative pain and the hip joint function changing situation, both the VAS score and the Harris score at every time point compared with, differences were statistically significant, which shows THRAt the treatment can relieve pain, improve the function of hip joint, and improve Harris score, especially in the 12 
months after surgery to improve the most obvious. But after 24 months, score index improvement is not obvious or lower, compared with 12 months score, difference was not statistically significant, no change or deterioration during this period (Table 2).

By comparing X-ray stage before and after operation, the follow-up time and Table 2, five cases worsened to Stage IIC from Stage IIB 24 months after the operation, of which four cases worsen to Stage III from Stage IIC; six cases worsen to Stage III from Stage IIC; THRA was performed.

The rest periods cases X-ray performance before and after operation stages basically stable, illustrate the hip joint without degradation, lesions in good repair and femoral head with no collapse (Table 3).

According to the etiology of variance analysis and II period lesions hip Kaplan-Meier survival curve (Figure 4), end to THRA, survival rates were $97.9 \%$ at 6 months ( $95 \% \mathrm{ci}$, 95\% - 95.8\%), 12 months was $98.5 \%$ (95\% ci, 96.2\% - 100.0\%), 24 months was $87.6 \%$ (95\% ci, $95 \%-81.4 \%$ ), and 36 months at $78.4 \%$ (95\% ci, $95 \%$ to $64.2 \%$ ) (Figure 5 ). 36 months survival rate decreased when the reason for the original IIB stage 5 cases of postoperative progress is IIC, 4 cases of IIC stage progress for III and THRA; The 6 cases of original IIC stage progress to III and THRA (Table 3), the 10 cases were glucocorticoid-induced ONFH, suggesting triggers associated with hormone application II lesions 3-year survival rate lowest (64.2\%), caused by other causes II period lesions survival satisfaction. 36 months follow-up after surgery according to the results, $79.72 \%$ of the total period, IIA improvement rate (93.33\%) was the best, IIB period improvement rate was $82.35 \%$, IIC improvement rate $(68 \%)$ was the lowest, no change and increase of cases are patients with glucocorticoid-induced ONFH (Table 4). It was further clarified that this method is best for Stage IIA and IIB ONFH in the aspects of effect improving and preventing the femoral head collapse to avoid THRA (Figure 6). The effect of phase II C though, but after 2 years of steroid ONFH risk of head collapse, and vascular steroid ONFH femoral head trabecular bone and bone marrow endothelial growth factor (vascular endothelial, growth factor, VEGF) and BMP content decreased significantly, and inhibited BMP synthesis of bone cells, the decrease of BMSC to the role of adipocyte differentiation and enhanced osteogenic differentiation. [12]. Therefore,

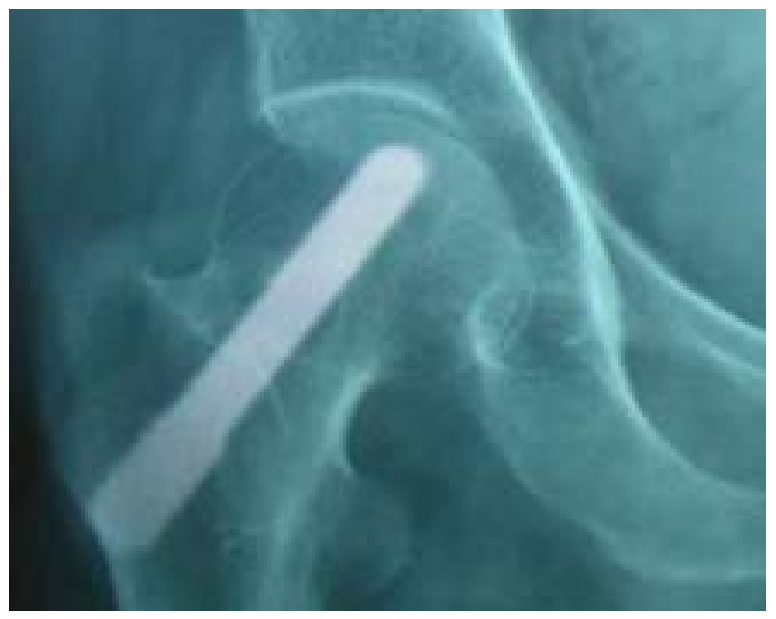

Figure 6. There was no collapse in the weight loading area of the femoral head and the Titanium rod had an excellent supporting effect when three years after the operation to recheck. 
hormone ONFH osteogenesis ability is weak, and accordingly is given priority to with bone resorption and granulation. The inhibitory effect of osteogenetic activity will continue to exist after surgery, namely "progressive osteonecrosis and bone loss", caused by internal fixation loosening [13]. Thus, this method should be performed prudently because of the poor effect for Stage IIC glucocorticoid-induced ONFH.

Although this study has a good result in the clinical, the operation cases are a little fewer, so a large sample research and long-term follow-up are needed.

\section{References}

[1] Scully, S.P., Aaron, R.K. and Urbaniak, J.R. (1998) Survival Analysis of Hips Treated with Core Decompression or Vascularized Fibular Grafting Because of Avascular Necrosis. Journal of Bone \& Joint Surgery-American Volume, 80, 1270-1275.

https://doi.org/10.2106/00004623-199809000-00004

[2] Mont, M.A., Carbone, J.J. and Fairbank, A.C. (1996) Core Decompression versus Nonoperative Management for Osteonecrosis of the Hip. Clinical Orthopaedics and Related Research, 324, 169-178. https://doi.org/10.1097/00003086-199603000-00020

[3] Gangji, V., Hauzeur, J.P., Matos, C., et al. (2004) Treatment of Osteonecrosis of the Femoral Head with Implantation of Autologous Bone-Marrow Cells. A Pilot Study. Journal of Bone \& Joint Surgery-American Volume, 86, 1153-1160. https://doi.org/10.2106/00004623-200406000-00006

[4] Siebenrock, K.A., Powell, J.N. and Ganz, R. (2010) Osteochondritis Dissecans of the Femoral Head. Hip International, 20, 489-496.

[5] Seyler, T.M., Cui, Q., Mihalko, W.M., Mont, M.A. and Saleh, K.J. (2007) Advances in Hip Arthroplasty in the Treatment of Osteonecrosis. Instructional Course Lectures, 56, 221-233.

[6] Yang, X.M., Shi, W., Du, Y.K., Huang, Y.P., Meng, X.Y., Yin, Y.L. and Li, H.G. (2008) Repairing Effect Comparison of Bone Marrow Stromal Cell, Osteoinductive Absorbing Material and Their Complexes on Rabbits with Early Avascular Necrosis of the Femoral Head. The Journal of Practical Medicine, 24, 2397-2400.

[7] Yang, X.M., Shi, W., Du, Y.K., Huang, Y.P., Meng, X.Y., Yin, Y.L. and Li, H.G. (2008) The Tissue Engineering Complexes of Autologous BMSC's Repairing Effect on the Rabbit's ANFH. Orthopaedic Biomechanics Materials and Clinical Study, 5, 1-5.

[8] ARCO (Association Research Circulation Osseous) Committee on Terminology and Classification (1992) ARCO News, 4, 41-46.

[9] Aldridge 3rd, J.M. and Urbaniak, J.R. (2004) Avascular Necrosis of the Femoral Head: Etiology, Pathop Hysiology, Classification, and Current Treatment Guidelines. The American Journal of Orthopedics, 33, 327-332.

[10] Yang, X.M., Shi, W., Du, Y.K., Huang, Y.P., Meng, X.Y., Yin, Y.L. and Li, H.G. (2009) Autologous Red Bone Marrow Tissue Engineering Complex Combine with Bouche Decompression and Implant of Hollow Titanium Screws for the Treatment of ANFH. Journal of Practical Orthopaedics, 15, 582-585.

[11] Yang, X.M., Shi, W., Meng, X.Y., Yin, Y.L., Huang, Y.P. and Li, H.G. (2009) Comparison of the Clinical Results of Core Decompression, Autologous Red Bone Marrow Tissue Engineered Bone with or without the Use of Titanium Cannulated Screws in Treating Early Necrosis of the Femoral Head. Journal of Clinical Orthopaedics, 12, 604-608.

[12] Li, X.D., Jin, L., Cui, Q.J., Wang, G.-J. and Balian, G. (2005) Steroid Effects on Osteogenesis through Mesenchymal Cell Gene Expression. Osteoporosis International, 16, 101-108. https://doi.org/10.1007/s00198-004-1649-7 
[13] Goffin, E., Baertz, G. and Rombouts, J.J. (2006) Long-Term Survivorship Analysis of Cemented Total Hip Replacement (THR) after Avascular Necrosis of the Femoral Head in Renal Transplant Recipients. Nephrology Dialysis Transplantation, 21, 784-788.

https://doi.org/10.1093/ndt/gfi233

Submit or recommend next manuscript to SCIRP and we will provide best service for you:

Accepting pre-submission inquiries through Email, Facebook, LinkedIn, Twitter, etc. A wide selection of journals (inclusive of 9 subjects, more than 200 journals)

Providing 24-hour high-quality service

User-friendly online submission system

Fair and swift peer-review system

Efficient typesetting and proofreading procedure

Display of the result of downloads and visits, as well as the number of cited articles

Maximum dissemination of your research work

Submit your manuscript at: http://papersubmission.scirp.org/

Or contact ss@scirp.org 